\title{
Synthesis of new spiroheterocycles-fused isoxazoline from 2-arylidenes-3-phenyl-1-indanones through a regio-and diastereospecific 1,3-dipolar cycloaddition
}

\author{
Asmae Mahfoud ${ }^{1}$, Abdelali Kerbal ${ }^{1}$, Nakkabi asmae ${ }^{2}$, Mohamed El Yazidi ${ }^{1}$, Mohamed Akhazzane ${ }^{3}$, \\ Mohamed Bakhouch ${ }^{4}$, Youssra Kanzouai ${ }^{1}$, Rachid Bouzammit ${ }^{1}$ and Ghali Al Houari ${ }^{1}$ \\ ${ }^{1}$ Engineering Laboratory of Organometallic and Molecular Materials and Environment, Faculty of Sciences \\ Dhar EL Mahraz, University Sidi Mohamed Ben Abdellah, P.O. Box 1796 (Atlas), 30000 Fes, Morocco \\ ${ }^{2}$ Laboratoire de chimie des materiaux et biotechnologie des produits naturels, Faculté des sciences, Unversité \\ Moulay Ismail, BP11201, 50000, Meknes, Morocco \\ ${ }^{3}$ Cité d'innovation, Sidi Mohamed Ben Abdellah University, Fez, Morocco \\ ${ }^{4}$ Department of Chemistry, Faculty of Sciences, Chouaib Doukkali University, P.O. Box 20, 24000, El Jadida, \\ Morocco
}

\begin{abstract}
New spiroisoxazolines 3 have been synthesized by 1,3-dipolar cycloaddition of arylnitrile oxides with 2-arylidenes-3-phenyl-1-indanones. The reaction occurs in a regiospecific and diastereospecific manner and leads to one cycloadduct in all the cases. The proposed structure of the obtained cycloadducts was established based on spectroscopic data and confirmed by radiocrystallographic study. The spectral data were in favor of the observed regiochemistry and diastereoselectivity of this reaction.
\end{abstract}

Keywords: Spiroisoxazoline; 2-arylidenes-3-phenyl-1-indanones; 1,3-dipolar cycloaddition; regioselectivity; stereoselectivity.

\section{Introduction}

Organic compound-fused 2-isoxazoline frameworks are a very interesting heterocycle in both organic and medicinal chemistry ${ }^{1-8}$. Isoxazolines also serve as essential building blocks for synthesizing a wide range of biologically active compounds ${ }^{9,10}$. For these reasons, a great deal of work has been devoted to synthesizing these kinds of heterocycles ${ }^{11,12}$.

In addition, the spiroisoxazoline scaffold forms an integral part of many beneficial biologically active compounds. Indeed, spiroisoxazolines exhibit an extensive array of biological and pharmacological activities such as antiviral ${ }^{13}$, anticancer 14,17,18 antimalarial ${ }^{15}$, antituberculosis ${ }^{16}$, antibacterial ${ }^{17,19}$ and antiparasitic ${ }^{20}$.

Spiroisoxazolines synthesized in our laboratory in 2005 have been patented for their antituberculosis and anti-HIV properties ${ }^{21,22}$. Since then, we have been further developing these highly promising molecules 16,20 .

In the current work, we used 2-arylidenes-3-phenyl1-indanones to synthesize spiroisoxazolines by reacting with arylnitrile oxide.The introduction of a phenyl group at the 3-position of the (E)-2-

*Corresponding author: Ghali Al Houari

Email address: ghalialhouari@gmail.com

DOI: http://dx.doi.org/10.13171/mjc02105141076gh (4-arylidene)-indanones made the reaction of 1,3dipolar cycloaddition with arylnitrile oxides not only regiospecific, but also stereospecific, and only the cycloadduct "anti" was obtained, making this a more efficient method.

\section{Results and Discussion}

The 1,3-dipolar cycloaddition reaction of alkynes/alkenes with nitrile oxides, respectively, constitutes a versatile tool to access isoxazole and isoxazoline heterocycles ${ }^{23-25}$. In this context, and in continuation of our ongoing research devoted to synthesis heterocyclic compounds with promising biological and pharmacological activities via 1,3dipolar cycloaddition across dipolarophiles with exocyclic double bond with several 1,3-dipoles ${ }^{16,20}$. We describe a reaction between arylnitrile oxides precursors (arylaldoximes) and arylidenes-indanone derivatives in one pot, to give the desired spiroisoxazolines 3 (Scheme 1). We carried out the reaction with 2-arylidenes-3-phenyl-1-indanones and a slight excess of arylnitrile oxide precursors in chloroform at a temperature not exceeding $5^{\circ} \mathrm{C}$ (Scheme 1). Arylnitrile oxides were generated

Received January 2, 2021

Accepted February 6, 2021

Published May 14, 2021 
"in situ" from corresponding arylaldoximes by action of sodium hypochlorite at low temperature so there is no dimerization of the dipole to furoxane. There are many ways to generate arylnitrile oxide. The most commonly used ones are dehydration of nitro compounds ${ }^{26}$ or the action of triethylamine on hydroxamoyl chlorides ${ }^{27}$. We chose to generate our arylnitrile oxide "in situ" by the action sodium hypochlorite on arylaldoximes because of its simplicity ${ }^{28}$. The 2-arylidenes-3-phenyl-1-indanones used as starting materials in this work were obtained through a Knoevenagel condensation of appropriate aldehyde on 3-phenyl-1-indanone in the presence of a basic medium, according to previously published protocol 29,30 .

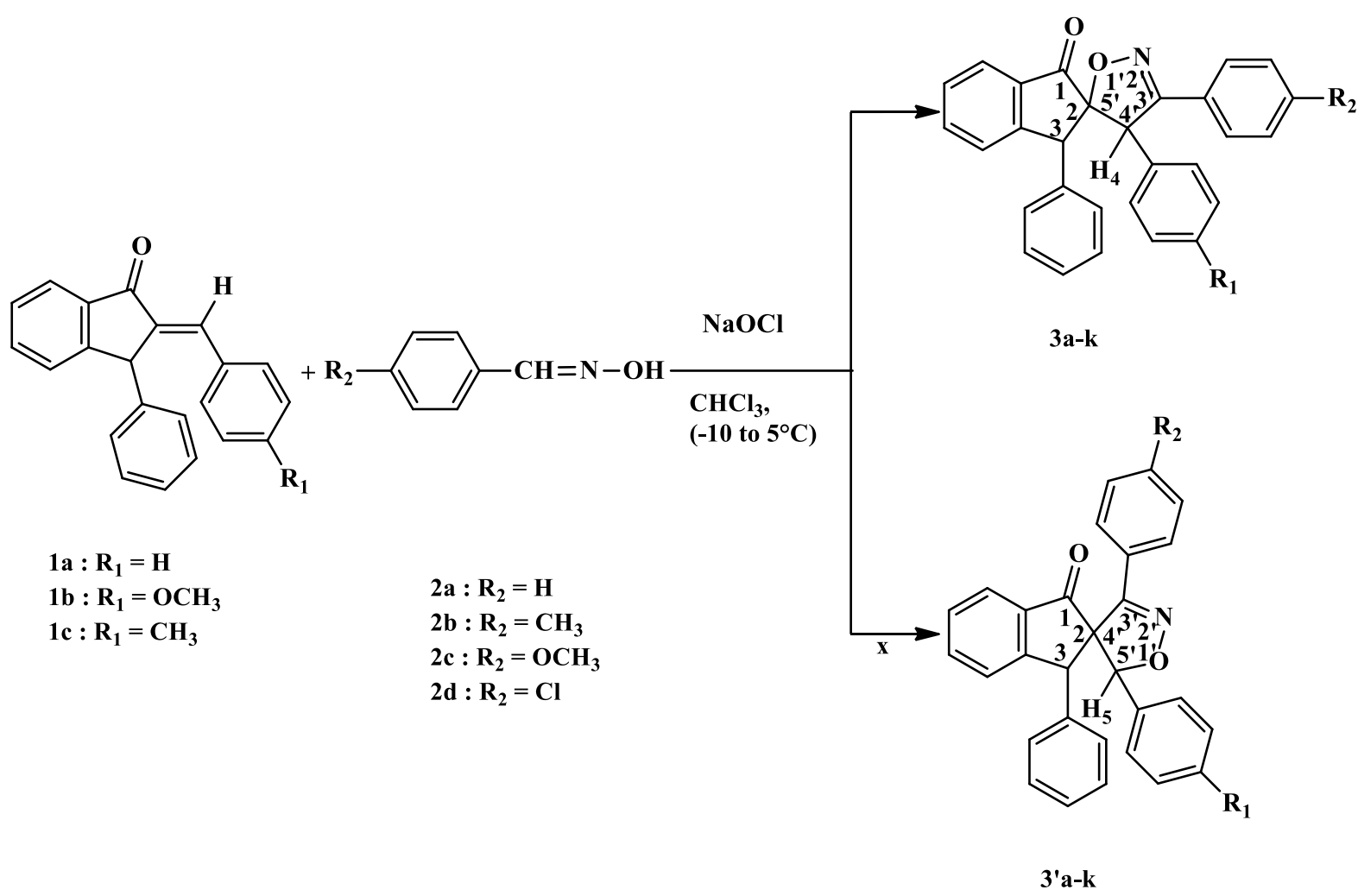

Scheme 1. Synthesis of spiroisoxazolines 3 (regiospecificity of the reaction)

NMR spectra of the crude product revealed the formation of only the regioisomer 3-phenyl-3',4'diaryl-4'H-spiro[indene-2,5'-isoxazol]-1(3H)-ones 3 (Scheme 1). The regiochemistry shown in this reaction was similar to the one obtained in reactions using other dipolarophiles ${ }^{31-37}$.

The structural assignment of the isolated cycloadducts 3 was made based on spectroscopic data (IR, NMR), which are summarized in the experimental part. The isolated cycloadducts were considered to be regioisomers of type $\mathbf{3}$, not regioisomers of type $\mathbf{3}$ '. This was based on the chemical shifts of the isoxazoline ring protons $\left(\mathrm{H}_{4}\right)$ compared to analogous cycloadducts. Thus the isoxazoline ring protons of $\mathbf{3}$ resonate at $4.4 \mathrm{ppm}$. In contrast, the isoxazoline ring protons $\mathrm{H}_{5}$, of cycloadducts 3 ' would be expected at higher chemical shift values due to the effect of oxygen atom compared with analogous cycloadducts ${ }^{38}$.

Moreover, the ${ }^{13} \mathrm{C}$ NMR spectrum reveals two characteristic signals at $199 \mathrm{ppm}$ and $53 \mathrm{ppm}$, corresponding to $\mathrm{C}=\mathrm{O}$ carbon and benzylic carbon $\mathrm{C}_{4}$. Furthermore, the signal of spiranic carbon $\left(\mathrm{C}_{2: 5}{ }\right)$ appears at $96 \mathrm{ppm}$, which agrees with the literature for the proposed regiochemistry ${ }^{39}$. If we suppose that $\mathbf{3}^{\prime}$ compounds are obtained, the spirannic carbon $\left(\mathrm{C}_{2: 4^{\prime}}\right)$ would have appeared at a lower shift since it would be far from the oxygen atom. These spectral data are in favor of regioisomer $\mathbf{3}$ and confirm the regiospecificity of the action of arylnitrile oxides on 2-arylidene-3-phenyl-1-indanones. In addition, the FT-IR spectrum of the cycloadduct $\mathbf{3}$ shows two significant bands around $1700 \mathrm{~cm}^{-1}$ and $1600 \mathrm{~cm}^{-1}$ assigned, respectively, to $\mathrm{C}=\mathrm{O}$ and $\mathrm{C}=\mathrm{N}$ stretching. We also noticed in most cases that the yield of the reaction is higher when dipole $\mathbf{2 a}(\mathrm{R}=\mathrm{H})$ is used. This can be explained by the less steric hindrance.

In the ${ }^{1} \mathrm{H}$ NMR spectrum of cycloadduct $\mathbf{3 b}$ (Figure 1), the signal of the methyle appears as a singlet at $2.28 \mathrm{ppm}$, while the signals of $\mathrm{H}_{4}$, and $\mathrm{H}_{3}$ appear at singlets at 4.42 and $4.75 \mathrm{ppm}$, respectively. The signals of the aromatic protons appear as multiplet between 6.36 and $7.95 \mathrm{ppm}$. 


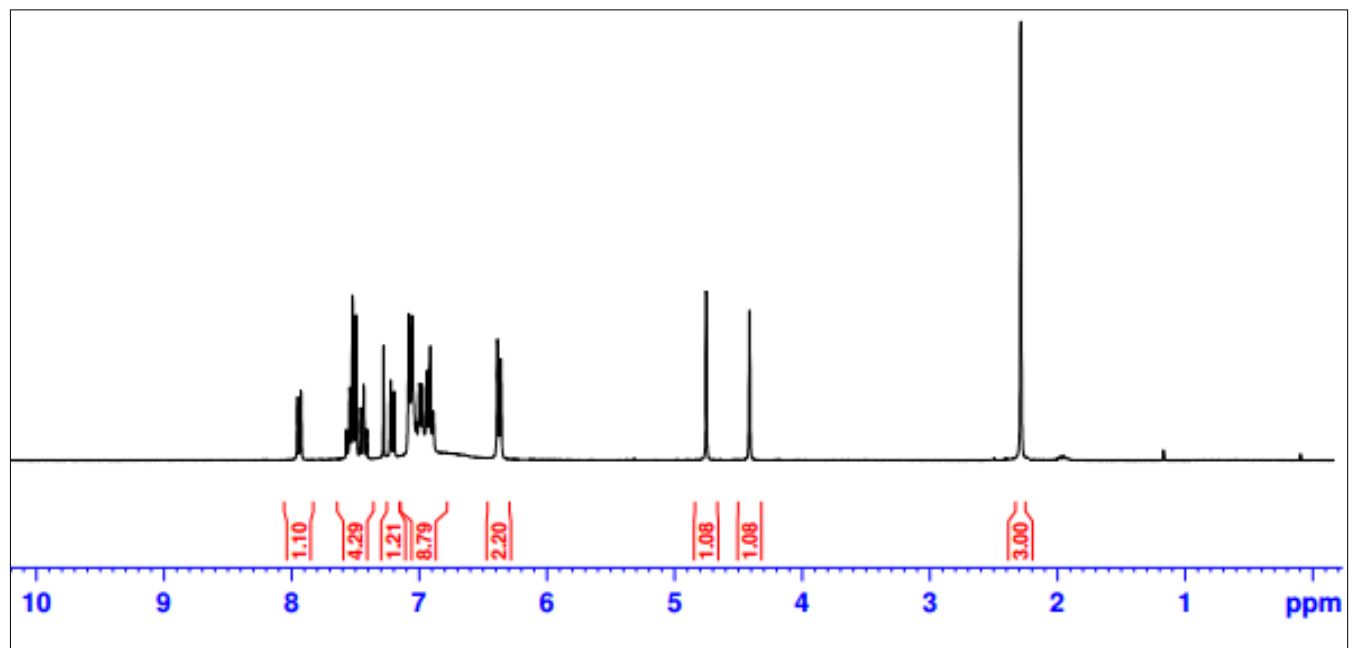

Figure 1. ${ }^{1} \mathrm{H}$ NMR spectrum of $\mathbf{3 b}\left(\mathrm{CDCl}_{3}\right)$

The stereoselectivity of 1,3-dipolar cycloaddition reactions is well known. This reaction is accompanied by the creation of one or two centers of chirality on the dipolarophile. If both faces of the latter are equivalent, the dipole can react from both sides of the dipolarophile with an equal probability, and then a racemic mixture is obtained. Diastereoselectivity is possible if both sides of the dipolarophile are diastereotopic. Each of the two possible approaches (syn and anti-approaches) would lead to a different diastereoisomer since the reaction studied here is regiospecific. Obtaining a single cycloadduct in all cases, while both approaches are theoretically possible, makes it possible to affirm that the reaction is diastereospecific. The dipole approach toward the dipolarophile is always from the opposite side of the phenyl substituent of the dipolarophile (Scheme 2).<smiles>[R]c1ccc(/C=C2/C(=O)c3ccccc3C2c2ccccc2)cc1</smiles>

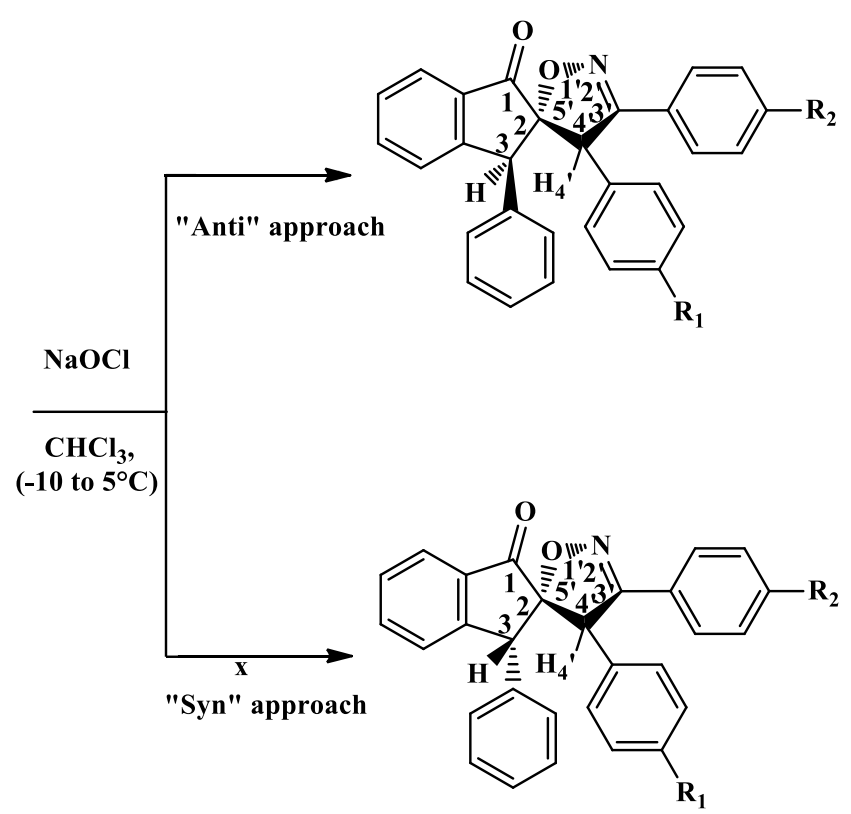

Scheme 2. Diastereospecifity of the reaction

The structure of cycloadduct $\mathbf{3 b}$, whose crystallographic data have been previously published ${ }^{31}$, was corroborated by X-ray single-crystal diffraction (Figure 2). This structural determination confirms the resulting regiospecificity and diastereospecificity. 


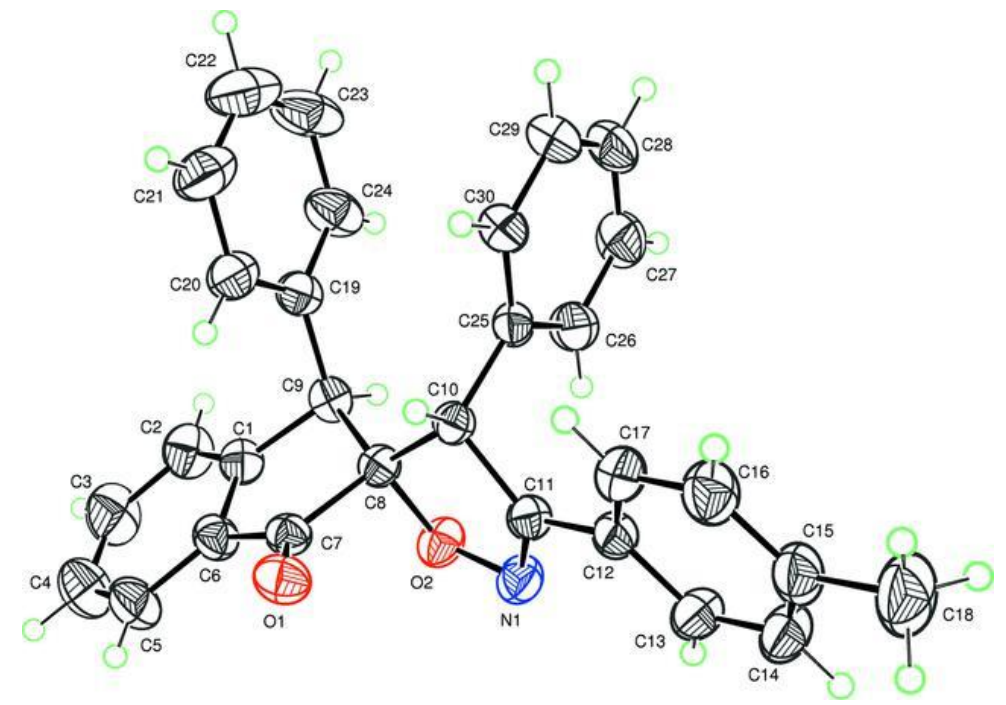

Figure 2. ORTEP diagram of cycloadduct $3 b^{*}$

*The molecular structure of the title compound with the atom labeling scheme. Displacement ellipsoids are drawn at the $50 \%$ probability level. $\mathrm{H}$ atoms are represented as small circles.

\section{Conclusion}

In summary, we have shown in this work that the spiro-isoxazolines 3 can easily be prepared by 1,3dipolar cycloaddition of 2-arylidene-3-phenyl-1indanones $\mathbf{1}$ with the appropriate arylnitriloxyde. We have also shown that these reactions were regiospecific and diastereospecific, due to the influence of steric factors, which are important in the stereoselectivity of the dipole-dipolarophilic approach. The high diastereospecificity and regiospecificity were determined based on spectroscopic data and corroborated by radiocrystallographic study.

\section{Acknowledgements}

The authors would like to thank the City of Innovation staff, Sidi Mohammed Ben Abdellah University-Fez, Morocco, for spectroscopic facilities.

\section{Experimental}

\subsection{General}

Chemicals and reagents were purchased from commercial sources. All melting points were determined using a Stuart SMP 10 melting point apparatus. The infrared spectra were recorded at room temperature using BRUKER VERTEX 70 spectrometer. The ${ }^{1} \mathrm{H}$ and ${ }^{13} \mathrm{C}$ NMR spectra were recorded at room temperature on BRUKER AVANCE II $300 \mathrm{MHz}$ instrument. Arylaldoximes and 2-arylidene-3-phenyl-1-indanones were prepared using previously reported procedures ${ }^{38,40}$.

\subsection{General procedure of the preparation of spiro-isoxazolines: 3}

In a $100 \mathrm{ml}$ flask, (2E)-2-benzylidene-3-phenyl-2,3dihydro-1H-inden-1-one ( $2 \mathrm{mmol}$ ) and appropriate arylaldoxime $(2.4 \mathrm{mmol})$ were dissolved in chloroform $(20 \mathrm{ml})$. The mixture was stirred at $263 \mathrm{~K}$ in an ice-brine bath, then $15 \mathrm{ml}$ of sodium hypochlorite $(\mathrm{NaOCl})$ were added dropwise, keeping the mixture below $278 \mathrm{~K}$. The mixture was left under magnetic stirring for 16 hours at room temperature until completion of the reaction. The mixture was washed with water until neutral $\mathrm{pH}$ and dried over anhydrous sodium sulfate. The solvent was evaporated under vacuum, and the obtained crude oil was crystallized in ethanol to obtain the spirocycloadducts 3 .

\subsection{Characterization of compounds} 3,3',4'-triphenyl-4'H-spiro[indene-2,5'-isoxazol]1(3H)-one (3a)

White solid: yield $=85 \%$; Mp. $=296^{\circ} \mathrm{C}$.

${ }^{1}$ H NMR (300 MHz, CDCl 3 ) (ס/ppm): 4.41(s, $\left.{ }_{4}{ }^{\prime}\right)$; 4.76(s, $\left.\mathrm{H}_{3}\right) ; 6.35-7.95(\mathrm{~m}, \mathrm{H}$. aromatic).

IR $(\mathrm{KBr}) \mathbf{c m}^{-1}: \mathbf{v}(\mathbf{C}=\mathbf{O})=1711 ; \mathrm{v}(\mathrm{C}=\mathrm{N})=1596$.

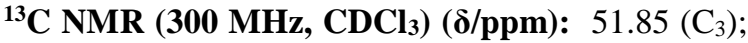
$53.61\left(\mathrm{C}_{4}\right) ; 96.08\left(\mathrm{C}_{2: 5}\right) ; 125.19 ; 126.25 ; 126.40$; $127.12 ; 127.28 ; 127.56 ; 128.46 ; 128.60 ; 130.13$; $132.81 ; 134.59 ; 135.96 ; 140.22 ; 155.33 ; 162.20$; $199.26(\underline{\mathrm{C}}=\mathrm{O})$.

\section{3-4'-diphenyl-3'-(p-tolyl)-4'H-spiro[indene-2,5'- isoxazol]-1(3H)-one (3b)}

White solid: yield $=70 \%$; $\mathrm{Mp} .=266^{\circ} \mathrm{C}$.

IR $(\mathrm{KBr}) \mathbf{c m}^{-\mathbf{1}}: \mathbf{v}(\mathbf{C}=\mathbf{O})=1712 ; \boldsymbol{v}(\mathbf{C}=\mathrm{N})=1597$.

${ }^{1}$ H NMR (300MHz, CDCl3) (ס/ppm): 2.28(s, 3H); 4.41(s, $\left.\mathrm{H}_{4}\right) ; 4.75\left(\mathrm{~s}, \mathrm{H}_{3}\right) ; 6.36-7.95(\mathrm{~m}, \mathrm{H}$. aromatic).

${ }^{13} \mathrm{C}$ NMR (300 MHz, $\left.\mathrm{CDCl}_{3}\right) \quad(\delta / \mathbf{p p m})$ : $21.34\left(\mathrm{C}_{(\mathrm{CH} 3)}\right) ; 51.93\left(\mathrm{C}_{3}\right) ; 53.74\left(\mathrm{C}_{4}\right) ; 95.91\left(\mathrm{C}_{2: 5}\right)$; $125.12 ; 125.65 ; 126.20 ; 126.36 ; 127.03 ; 127.31$; $127.49 ; 128.38 ; 128.45 ; 128.52 ; 129.19 ; 132.93$; 134.77; $135.82 ; \quad 140.31 ; \quad 155.33 ; \quad 162.16$; $199.22(\underline{\mathrm{C}}=\mathrm{O})$. 
3'-(4-methoxyphenyl)-3,4'-diphenyl-4'Hspiro[indene-2,5'-isoxazol]-1(3H)-one (3c) White solid; yield $=75 \%$; Mp. $=258^{\circ} \mathrm{C}$.

IR $(\mathrm{KBr}) \mathbf{c m}^{-1}: \mathbf{v}(\mathbf{C}=\mathbf{O})=1712 ; \mathbf{v}(\mathbf{C}=\mathrm{N})=1597$. ${ }^{1} \mathbf{H}$ NMR (300MHz, $\left.\mathbf{C D C l}_{3}\right)$ ( $\left.\mathbf{\delta} / \mathbf{p p m}\right): 3.75(\mathrm{~s}, 3 \mathrm{H})$; 4.40( $\left(\mathrm{s}, \mathrm{H}_{4}\right)$; $4.73\left(\mathrm{~s}, \mathrm{H}_{3}\right) ; 6.36-7.95(\mathrm{~m}, \mathrm{H}$. aromatic).

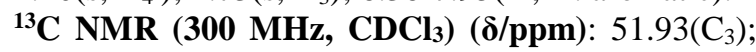
53.84( $\left(\mathrm{C}_{4}\right) ; \quad$ 55.21 $\left(\mathrm{C}_{(\mathrm{OCH} 3)}\right) ; \quad 95.82\left(\mathrm{C}_{2: 5},\right) ; 113.98$; $121.00 ; 125.12 ; 126.20 ; 126.36 ; 127.03 ; 127.32$; $128.37 ; 128.46 ; 128.54 ; 129.10 ; 132.95 ; 134.84$; $135.81 ; \quad 140.33 ; \quad 155.34 ; \quad 161.05 ; 161.80$; 199.29( $\mathrm{C}=\mathrm{O})$

\section{3'-(4-chlorophenyl)-3,4'-diphenyl-4'H-} spiro[indene-2,5'-isoxazol]-1(3H)-one (3d) White solid; yield $=80 \%$; Mp. $=228^{\circ} \mathrm{C}$.

IR $(\mathrm{KBr}) \mathbf{c m}^{-1}: \mathbf{v}(\mathbf{C}=\mathbf{O})=1712 ; \mathrm{v}(\mathbf{C}=\mathrm{N})=1594$.

${ }^{1}$ H NMR (300MHz, CDCl 3$)$ ( $\left.\delta / \mathbf{p p m}\right): 4.42\left(\mathrm{~s}, \mathrm{H}_{4}{ }^{\prime}\right)$; 4.72(s, $\left.\mathrm{H}_{3}\right) ; 6.31-7.96(\mathrm{~m}, \mathrm{H}$. aromatic)

${ }^{13} \mathrm{C}$ NMR (300 MHz, CDCl 3$)$ ( $\left.\delta / \mathbf{p p m}\right): 51.85\left(\mathrm{C}_{3}\right)$; $53.58\left(\mathrm{C}_{4}\right) ; 96.25\left(\mathrm{C}_{2: 5}\right) ; 125.17 ; 126.30 ; 126.39$; $127.01 ; \quad 127.28 ; \quad 128.51 ; 128.68 ; 128.75$; $128.81 ; 132.77 ; 134.31 ; 135.99 ; 136.12 ; 140.12$; $155.30 ; 161.27 ; 199.07(\underline{\mathrm{C}}=\mathrm{O})$.

4'-(4-methoxyphenyl)-3,3'-diphenyl-4'Hspiro[indene-2,5'-isoxazol]-1(3H)-one (3e) White solid; yield $=70 \%$; Mp. $=260^{\circ} \mathrm{C}$.

IR $(\mathbf{K B r}) \mathbf{c m}^{-1}: \mathbf{v}(\mathbf{C}=\mathbf{O})=1715 ; \mathbf{v}(\mathbf{C}=\mathbf{N})=1603$.

${ }^{1}$ H NMR (300MHz, $\left.\mathbf{C D C l}_{3}\right)$ ( $\left.\mathbf{6} / \mathbf{p p m}\right): 3.74$ (s, 3H); $4.39\left(\mathrm{~s}, \mathrm{H}_{4}\right)$ ); $4.72\left(\mathrm{~s}, \mathrm{H}_{3}\right) ; 6.40-7.55$ (m, H. aromatic)

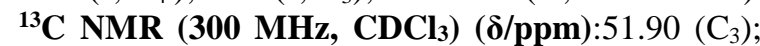
$52.89\left(\mathrm{C}_{4}\right) ; 55.26\left(\mathrm{C}_{(\mathrm{OCH} 3)}\right) ; 95.98\left(\mathrm{C}_{2: 5}\right)$;

$114.08 ; 125.15 ; 126.33 ; 126.41 ; 126.64 ; 127.28$; $127.57 ; 128.44 ; 128.49 ; 130.08 ; 132.92 ; 135.92$; $140.43 ; 155.33 ; 158.73 ; 162.25 ; 199.48(\mathrm{C}=\mathrm{O})$.

4'-(4-methoxyphenyl)-3-phenyl-3'-(p-tolyl)-4'Hspiro[indene-2,5'-isoxazol]-1(3H)-one (3f) White solid; yield $=80 \%$; Mp. $=292^{\circ} \mathrm{C}$.

IR $(\mathrm{KBr}) \mathbf{c m}^{-1}: \mathrm{v}(\mathbf{C}=\mathrm{O})=1713 ; \mathrm{v}(\mathbf{C}=\mathrm{N})=1603$.

${ }^{1}$ H NMR (300MHz, CDCl 3 ) (ס/ppm): 2.29(s, 3H); 3.73(s, 3H); 4.38(s, $\mathrm{H}_{4}$ ) ; 4.71( $\left(\mathrm{s}, \mathrm{H}_{3}\right)$; 6.39-7.56 (m, H. aromatic)

${ }^{13}$ C NMR (300 MHz, $\left.\mathbf{C D C l}_{3}\right)$ ( $\left.\mathbf{\delta} / \mathbf{p p m}\right): 21.41\left(\mathrm{C}_{(\mathrm{CH} 3)}\right)$; $51.92\left(\mathrm{C}_{3}\right) ; 52.93\left(\mathrm{C}_{4}\right) ; 55.25\left(\mathrm{C}_{(\mathrm{OCH} 3)}\right) ; 95.81\left(\mathrm{C}_{2: 5}\right)$; $114.04 ; 125.13 ; 125.63 ; 126.30 ; 126.40 ; 126.80$; $127.29 ; 127.51 ; 128.39 ; 128.43 ; 129.22 ; 132.97$; $135.87 ; 140.33 ; 140.48 ; 155.34 ; 158.67 ; 162.21$; 199.48( $\mathrm{C}=\mathrm{O})$.

3',4'-bis(4-methoxyphenyl)-3'-phenyl-4'Hspiro[indene-2,5'-isoxazol]-1(3H)-one (3g) White solid; yield $=79 \%$; Mp. $=279^{\circ} \mathrm{C}$.

IR $(\mathrm{KBr}) \mathbf{c m}^{-1}: \mathrm{v}(\mathrm{C}=\mathrm{O})=1710 ; \mathrm{v}(\mathrm{C}=\mathrm{N})=1605$.

${ }^{1}$ H NMR (300MHz, CDCl 3$)$ (ס/ppm): 3.74(s, 3H); 3.76(s, 3H); 4.38(s, $\mathrm{H}_{4}$ ) ; 4.68(s, $\left.\mathrm{H}_{3}\right)$; 6.39-7.94 (m, H. aromatic)

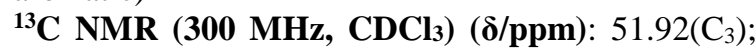
53.05( $\left.\mathrm{C}_{4}\right) ; \quad 55.26\left(\mathrm{C}_{(\mathrm{OCH} 3)}\right) ; \quad 95.72\left(\mathrm{C}_{2: 5}\right) ; 120.97$; $1254.12 ; 126.30 ; 126.40 ; 126.87 ; 127.29 ; 128.39$; $128.42 ; 129.12 ; 132.98 ; 135.86$;
140.49; $155.34 ; \quad 158.67 ; \quad 160.98 ; \quad 161.85 ;$ 199.49( $\underline{\mathrm{C}}=\mathrm{O})$.

3'-(4-chlorophenyl)-4'-(4-methoxyphenyl)-3phenyl-4'H-spiro[indene-2,5' - isoxazol]-1(3H)-one (3h)

White solid; yield $=76 \%$; $\mathrm{Mp} .=281{ }^{\circ} \mathrm{C}$.

IR $(\mathrm{KBr}) \mathbf{c m}^{-1} \mathbf{v} \mathbf{v}(\mathbf{C}=\mathbf{O})=1714 ; \boldsymbol{v}(\mathbf{C}=\mathbf{N})=1595$.

${ }^{1}$ H NMR (300MHz, CDCl $)$ ( $\left.\mathbf{6} / \mathbf{p p m}\right): 3.74(\mathrm{~s}, 3 \mathrm{H})$; 4.39(s, $\left.\mathrm{H}_{4}\right)$; 4.68( $\left(\mathrm{s}, \mathrm{H}_{3}\right) ; 6.38-7.95(\mathrm{~m}, \mathrm{H}$. aromatic)

${ }^{13} \mathrm{C}$ NMR (300 MHz, $\left.\mathrm{CDCl}_{3}\right)$ ( $\left.\mathbf{\delta} / \mathbf{p p m}\right): 51.85\left(\mathrm{C}_{3}\right)$; $52.80\left(\mathrm{C}_{4}\right) ; \quad 55.27\left(\mathrm{C}_{(\mathrm{OCH} 3)}\right) ; \quad 95.82\left(\mathrm{C}_{2: 5},\right) ; 113.97$; 114.17 ; '126.29; 127.01; 127.27; 129.74; 132.82; $133.45 ; 136.03 ; 136.07 ; 140.30$;

$155.31 ; 158.84 ; 161.31 ; 199.30(\underline{\mathrm{C}}=\mathrm{O})$.

3,3'-diphenyl-4' -(p-tolyl)-4'H-spiro[indene-2,5' isoxazol]-1(3H)-one (3i)

White solid; yield $=88 \%$; Mp. $=264^{\circ} \mathrm{C}$.

IR $(\mathrm{KBr}) \mathbf{c m}^{-1}: \mathbf{v}(\mathbf{C}=\mathrm{O})=1713 ; \mathrm{v}(\mathrm{C}=\mathrm{N})=1604$.

${ }^{1}$ H NMR (300MHz, $\left.\mathbf{C D C l}_{3}\right)$ (ס/ppm): 2.24(s, 3H); $4.40\left(\mathrm{~s}, \mathrm{H}_{4}\right) ; 4.73\left(\mathrm{~s}, \mathrm{H}_{3}\right) ; 6.35-7.57(\mathrm{~m}, \mathrm{H}$. aromatic)

${ }^{13} \mathrm{C} \quad \mathrm{NMR} \quad\left(300 \quad \mathrm{MHz}, \mathrm{CDCl}_{3}\right) \quad(\delta / \mathbf{p p m})$ : $21.07\left(\mathrm{C}_{\left(\mathrm{CH}_{3}\right)}\right) ; 51.92\left(\mathrm{C}_{3}\right) ; 53.26\left(\mathrm{C}_{4}{ }^{\prime}\right) ;$ 95.81 $\left(\mathrm{C}_{2: 5}\right)$; $125.15 ; 126.24 ; 126.41 ; 127.33 ; 127.57 ; 128.31$; $128.42 ; 128.49 ; 129.25 ; 130.07 ; 131.50$;

135.92; 136.78; $140.31 ; \quad 155.58 ; \quad 157.97$; 201.27( $\underline{\mathrm{C}}=\mathrm{O})$.

3-phenyl-3',4'-di-p-tolyl-4'H-spiro[indene-2,5' isoxazol]-1(3H)-one (3j)

White solid; yield $=69 \%$; Mp. $=190^{\circ} \mathrm{C}$.

IR $(\mathrm{KBr}) \mathbf{c m}^{-1}: \mathbf{v}(\mathbf{C}=\mathbf{O})=1712 ; \mathbf{v}(\mathbf{C}=\mathrm{N})=1602$.

${ }^{1}$ H NMR (300MHz, $\mathbf{C D C l}_{3}$ ) ( $\left.\mathbf{\delta} / \mathbf{p p m}\right): 2.30(\mathrm{~s}, 3 \mathrm{H})$; $2.30(\mathrm{~s}, 3 \mathrm{H}) ; 4.39\left(\mathrm{~s}, \mathrm{H}_{4}\right)$; 4.71(s, $\left.\mathrm{H}_{3}\right) ; 6.35-7.96$ (m, H. aromatic).

${ }^{13} \mathrm{C} \quad$ NMR $\quad\left(300 \quad \mathrm{MHz}, \quad \mathrm{CDCl}_{3}\right)$

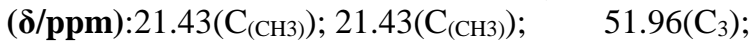
53.34 $\left(\mathrm{C}_{4}\right) ;$ 95.82 $\left(\mathrm{C}_{2: 5}\right) ; 124.21 ; 125.11 ; 125.89$; $126.19 ; 126.39 ; 126.92 ; 127.50 ; 127.59 ; 127.84$; $128.28 ; 128.37 ; 128.97 ; 129.21 ; 131.55 ; 134.90$; $135.74 ; 135.83 ; 194.69(\underline{\mathrm{C}}=\mathrm{O})$.

3'-(4-methoxyphenyl)-3-phenyl-4'-(p-tolyl)-4'Hspiro[indene-2,5'-isoxazol]-1(3H)-one (3k) White solid; yield $=65 \%$; $\mathrm{Mp} .=208^{\circ} \mathrm{C}$.

IR $(\mathbf{K B r}) \mathbf{c m}^{-1}: \mathbf{v}(\mathbf{C}=\mathbf{O})=1712 ; \mathbf{v}(\mathbf{C}=\mathbf{N})=1597$.

${ }^{1}$ H NMR (300MHz, CDCl $)$ ( $\left.\mathbf{6} / \mathbf{p p m}\right): 2.29$ (s, 3H); 3.76(s, 3H); 4.38(s, $\mathrm{H}_{4}$ ) ; 4.69(s, $\left.\mathrm{H}_{3}\right)$; 6.35-7.94 (m, H. aromatic)

${ }^{13} \mathrm{C} \quad \mathrm{NMR} \quad\left(300 \quad \mathrm{MHz}, \mathrm{CDCl}_{3}\right) \quad(\boldsymbol{\delta} / \mathbf{p p m})$ : $21.05\left(\mathrm{C}_{(\mathrm{CH} 3)}\right) ; 51.95\left(\mathrm{C}_{3}\right) ; 53.44\left(\mathrm{C}_{4}\right) ; 55.23\left(\mathrm{C}_{(\mathrm{OCH} 3)}\right)$; 95.82 $\left(\mathrm{C}_{2: 5}\right) ; 114.04 ; 125.13 ; 125.63 ; 126.30 ; 126.40$; $126.80 ; 127.29 ; 127.51 ; 128.39 ; 128.43 ; 129.22$; $132.97 ; 135.87 ; 140.33 ; 140.48 ; 155.34 ; 158.67$; $162.21 ; 194.69(\underline{\mathrm{C}}=\mathrm{O})$.

X-Ray cristallography for $3 \mathrm{~b}$ : $\left(3,4^{\prime}\right.$-Diphenyl-3'-ptolyl-4' $H$-spiro[indan-2,5'-[1,2] oxazol]-1-one) In the title compound, $\mathrm{C}_{3} \mathrm{OH}_{23} \mathrm{NO}_{2}$, the fivemembered rings are both in envelope conformations with the same spiro $\mathrm{C}$ atom as the flap. The benzene ring and the two phenyl rings are inclined to the mean 
plane of the indene ring system by 83.98 (8), 81.46 (8), and 72.31 (7) ${ }^{\circ}$. In the crystal, molecules are linked by pairs of $\mathrm{C}_{-} \mathrm{H}_{-}$_O $\mathrm{O}$ hydrogen bonds into inversion dimers. The dimers are further connected by C-H_ _Ninteractions, forming layers' parallel to (101).

\section{Data collection}

\section{Bruker X8 APEX diffractometer.}

38803 measured reflections, 5942 independent reflections, 3783 reflections with I > 2_(I), Rint $=0.042$

Crystal data

$\mathrm{Mr}=429.49$, Monoclinic, $\mathrm{P} 21=\mathrm{n}, \mathrm{a}=9.7381$ (7) $\AA$ $\mathrm{b}=20.5072$ (14) $\AA, \mathrm{c}=11.8261$ (8) $\AA$, b = 102.836 $(2)^{\circ}$,

$\mathrm{V}=2302.7$ (3) $\AA^{3}, \mathrm{Z}=4$, Mo K $\alpha$ radiation, $\mu=0.08$ $\mathrm{mm}^{-1}, \mathrm{~T}=296 \mathrm{~K}, 0.42 \times 0.31 \times 0.26 \mathrm{~mm}$.

Further crystallographic data can be obtained free of charge from the Cambridge Crystallographic Data Center through www.ccdc.cam.ac.uk/data_request/cif.CCDC 1431561

\section{References}

1- P. Dai, X. Tan, Q. Luo, X. Yu, S. Zhang, F. Liu, W. Zhang, Synthesis of 3-Acyl-isoxazoles and $\Delta^{2}$-Isoxazolines from Methyl Ketones, Alkynes or Alkenes, and tert-Butyl Nitrite via a Csp ${ }^{3}-\mathrm{H}$ Radical Functionalization/Cycloaddition Cascade, Org. Lett., 2019, 21 (13), 5096-5100.

2- M. Miglianico, M. Eldering, H. Slater, N. Ferguson, P. Ambrose, R. S. Lees, K. M. J. Koolen, K. Pruzinova, M. Jancarova, P. Volf, C. J. M. Koenraadt, H. Duerr, Gr. Trevitt, B. Yang, A. K. Chatterjee, J. Wisler, A. Sturm, T. Bousema, R. W. Sauerwein, P. G. Schultz, M. S. Tremblay, K. J. Dechering, Repurposing isoxazoline veterinary drugs for control of vector-borne human diseases, Proc. Natl. Acad. Sci USA, 2018, 115 (29), E6920-E6926.

3- M. Li, B. Song, M. Imerhasan, H. Youji, Progress in synthesis and bioactivity of spiroisoxazoline compounds, Chinese journal of organic chemistry, 2018, 38 (2), 378-400.

4- R. Sakly, H. Edziri, M. Askri, M. Knorr, K. Louven, C. Strohmann, M. Mastouri, Synthesis of New Spirooxindole-Fused Isoxazoline/Triazole and Isoxazoline/Isoxazole Derivatives from Three-Component 1,3-Dipolar Cycloaddition, Journal of Heterocyclic Chemistry, 2017, 54 (6), 3554-3564.

5- H. Deng, B. Zhang, Y. Xu, Y. Zhang, J. Huo, L. Zhang, G. Chang, A simple approach to prepare isoxazoline-based porous polymer for the highly effective adsorption of 2,4,6trinitrotoluene (TNT): Catalyst-free click polymerization between an in situ generated nitrile oxide with polybutadiene, Chemical Engineering Journal, 2020, 393, 124674.

6- C. C. Bernal, L. C. Vesga, S. C. MendezSánchez, Synthesis and anticancer activity of new tetrahydroquinoline hybrid derivatives tethered to isoxazoline moiety, Med. Chem. Res., 2020, 29, 675-689.

7- V. Palmieri, W. J. Dodds, J. Morgan, E. Carney, H. A. Fritsche, J. Jeffrey, R. Bullock, J. P. Kimball, Survey of canine use and safety of isoxazoline parasiticides, Vet. Med. Sci., 2020, 6 (4), 933-945.

8- N. Lamassiaude, B. Toubate, C. Neveu, P. Charnet, C. Dupuy, F. Debierre-Grockiego, I. Dimier-Poisson, C. L. Charvet, Distinct molecular targets for macrocyclic lactone and isoxazoline insecticides in the human louse: new prospects for the treatment of pediculosis, bioRxiv, 2020. doi: $10.1101 / 2020.08 .06 .239400$.

9- H. Abolhasani, S. Dastmalchi, M. HamzehMivehroud, B. Daraei, A. Zarghi, Derivatives as Selective Design, Synthesis and Biological Evaluation of New Tricyclic Spiroisoxazoline COX-2 Inhibitors and Study of Their COX-2 Binding Modes via Docking Studies, Medicinal Chemistry Research, 2016, 25 (5), 858-869.

10-S. K. Prajapti, S. Shrivastava, U. Bihade, A. K. Gupta, V. G. M. Naidu, U. C. Banerjee, B. N. Babu, Synthesis and Biological Evaluation of Novel $\Delta^{2}$-Isoxazoline Fused Cyclopentane Derivatives as Potential Antimicrobial and Anticancer Agents, Med.Chem.Comm., 2015, 6 (5), 839-845.

11-K. Kaur, V. Kumar, A. K. Sharma, G. K. Gupta, Isoxazoline Containing Natural Products as Anticancer Agents, European journal of medicinal chemistry, 2014, 77, 121-133.

12-G. Suresh, R. Venkata Nadh, N. Srinivasu, K. Kaushal, Novel Coumarin Isoxazoline Derivatives: Synthesis and Study of Antibacterial Activities, Synthetic Communications, 2016, 46 (24), 1972-1980.

13-P. Das, M. H. Hasan, D. Mitra, R. Bollavarapu, E. J. Valente, R. Tandon, D. Raucher, A. T. Hammell, Design, Synthesis, and Preliminary Studies of Spiro-isoxazoline-peroxides against Human Cytomegalovirus and Glioblastoma, J. Org. Chem., 2019, 84 (11), 6992-7006.

14-P. Das, A. O. Omollo, L. J. Sitole, E. McClendon, E. J. Valente, D. Raucher,L. R. Walker, A. T. Hammell, Synthesis and Investigation of Novel Spiro-isoxazolines as Anti-Cancer Agents, Tetrahedron Lett., 2015, 56 (14), 1794-1797.

15-S. Pratap, F. Naaz, S. Reddy, K. K. Jha, K. Sharma, D. Sahal, Anti-proliferative and antimalarial activities of spiroisoxazoline analogues of artemisinin, Arch. Pharm., 2019, 352 (1), 1800192 . 
16-G. Al Houari, A. Kerbal, B. Bennani, M. Filali. Baba, M. Daoudi, T. Ben Hadda, Drug design of new antitubercular agents:1,3-dipolar cycloaddition reaction of para-substitutedbenzadoximes and 3-para-methoxy-benzylidenisochroman-4-ones, ARKIVOC, 2008 (xii), 42-50.

17-M. Li, B. Song, I. Mukhtar, Progress in Synthesis and Bioactivity of Spiroisoxazoline Compounds, Chinese Journal of Organic Chemistry, 2018, 38 (2), 378-400.

18-A. Abolhasani, F. Heidari, S. Noori, S. Mousavi, H. Abolhasani, Cytotoxicity Evaluation of Dimethoxy and TrimethoxyIndanonic Spiroisoxazolines Against Cancerous Liver Cells, Current Chemical Biology, 2020, 14 (38). doi: 10.2174/2212796813666190926112807.

19-N. Najim, Y. Bathich, M. Zain, A. S. Hamzah, Z. Shaameri, Evaluation of the Bioactivity of Novel Spiroisoxazoline Type Compounds against Normal and Cancer Cell Lines, Molecules, 2010, 15 (12), 9340-9353.

20-T. Ben Hadda, A. Kerbal, B. Bennani, G. Al Houari, M. Daoudi, A. C. L. Leite, V. H. Masand, R. D. Jawarkar, Z. Charrouf, Molecular Drug Design, Synthesis and Pharmacophore Site Identification of Spiroheterocyclic Compounds: Trypanosoma Crusi Inhibiting Studies, Medicinal Chemistry Research, 2013, 22 (1), 57-69.

21-B. Bennani, A. Kerbal, N. Ben Larbi, T. BenHadda, Moroccan patent, 2005, OMPIC, $\mathrm{N}^{\circ}$ 27361.

22-B. Bennani, A. Kerbal, N. Ben Larbi, T. BenHadda, Moroccan patent, 2005, OMPIC, $\mathrm{N}^{\circ}$ 27362.

23- M. Zaki, A. Oukhrib, M. Akssira, S. BerteinaRaboin, Synthesis of novel spiro-isoxazoline and spiro-isoxazolidine derivatives of tomentosin, RSC Adv., 2017, 7, 6523-6529.

24-G. P. Savage, Spiro Isoxazolines via Nitrile Oxide 1,3-Dipolar Cycloaddition Reactions, Current Organic Chemistry, 2010, 14 (14), 1478-1499.

25-B. Mohon Chaki, K. Wakita, S. Takizawa, K. Takenaka, H. Sasai, Enantioselective Synthesis of Spiro (Isoxazole-Isoxazoline) Hybrid Ligand, heterocycles, 2018, 97 (1), 493-505.

26-T. Shimizu, Y. Hayashi, H. Shibafuchi, K. Teramura, A Convenient Preparative Method of Nitrile Oxides by the Dehydration of Primary Nitro Compounds with Ethyl Chloroformate or Benzenesulfonyl Chloride in the Presence of Triethylamine, Bull. Chem. Soc. Japan, 1986, 59, 2827 - 2831.

27- K. Liu, B. R. Shelton, R. K. Howe, A particularly convenient preparation of benzohydroximinoyl chlorides (nitrile oxide precursors), J. Org. Chem., 1980, 45, 3916 - 3918.

28-N. Naji, M. Soufiaoui, P. Moreau, Synthèse d'isoxazolines et d'isoxazoles F-alkylés en milieu biphasique $\mathrm{CHCl}_{3}-\mathrm{NaOCl}$, Journal of Fluorine Chemistry, 1996, 79 (2), 179-183.

29-S. Lafquih Titouani, M. Soufiaoui, L. Toupet, R. Carrié, Reaction de Diels Alder de l'isoprene et du dimethyl-2,3 butadiene avec quelques Arylidene-2 Indanones, Tetrahedron, 1990, 11, 3869-3878.

30-C. F. Koelsch, H. Hochmann, C. T. Le Claire, The Friedel-Crafts Reaction with Cinnamic, Crotonic, and $\beta$-Chlorocrotonic Acids, Journal of the American Chemical Society, 1943, 65 (1), 59-60.

31-A. Mahfoud, G. Al Houari, M. El Yazidi, M. Saadi, L. El Ammari, Crystal Structure of 3,4'-diphenyl-3'-p-tolyl-4'H-spiro-[Indan-2,5' [1,2]oxazol]-1-one, Acta Crystallographica, Section E, 2015, 71 (11), o873-o874.

32-M. Bakhouch, M. El Yazidi, G. Al Houari, M. Saadi, L. El Ammari, 3',4'-Diphenyl-3H,4'HSpiro-[Benzo[b]Thio-phene-2,5'-Isoxazol]-3-one, IUCrData, 2018, 3 (7), x181019.

33-M. Bakhouch, M. El Yazidi, G. Al Houari, M. Saadi, L. El Ammari, 3'-(4-Chlorophenyl)-4'Phenyl-3H ,4' H -spiro[benzo[b]thiophene-2,5'isoxazol]-3-one, IUCrData, 2017, 3 (5), x170677.

34-N. Mishriky, F. M. Asaad, Y. A. Ibrahim, A. S. Girgis, 1,3-Dipolar Cycloaddition of Arylnitrile Oxides to Some Exocyclic Alkenes and Regioselective Synthesis of Spiro Isoxazolines, Journal of Chemical Research, Synopses, 1997, 12, 438.

35-H. Yazdani, A. Bazgir, Lewis Acid-Catalyzed Regio- and Diastereoselective Synthesis of Spiroisoxazolines via One-Pot Sequential Knoevenagel Condensation/1,3-Dipolar Cycloaddition Reaction, Synthesis, 2019, 51 (7), 1669-1679.

36-X. Li, X. Yu, Y. Feng, Synthesis of novel bisspiroisoxazolines through 1,3-dipolar cycloaddition of nitrile oxide with $\alpha, \alpha$ bis(arylmethylidene)cycloalkanone, Chinese Journal of Chemistry, 2009, 27 (8), 1531-1536.

37-S. Boudriga, M. Askri, R. Gharbi, M. Rammah, K. Ciamala, 1,3-Dipolar cycloadditions of arylcarbonitrile oxides and diarylnitrilimines with some 2-arylmethylene 1,3-indanediones; regiochemistry of the reactions, Journal of Chemical Research, Synopses, 2003, 4, 204-207.

38-R. Chekti, M. Soufiaoui, L. Toupet, Régiochimie et diastéréochimie de l'addition des arylnitrile oxides sur les énones derivées d'indanones, Bulletin des Sociétés Chimiques Belge, 1991, 100 (2), 153-157.

39-N. G. Argyropoulos, E. Coutouli-Argyropoulou, 1,3-Dipolar Cycloaddition Reactions of 2Phenyl-4-Arylidene-5(4H)-Oxazolones with Nitrile Oxides, Journal of Heterocyclic Chemistry, 1984, 21 (5), 1397-1400.

40-C. Grundmann, R. Richter, Nitrile Oxides. IX. Basic Substituted Stable Nitrile Oxides, The Journal of Organic Chemistry, 1967, 32 (7), 2308-2312. 